University of Nebraska - Lincoln DigitalCommons@University of Nebraska - Lincoln

Mechanical \& Materials Engineering Faculty Mechanical \& Materials Engineering, Department

Publications

2011

\title{
Cohesive Zone Model to Predict Fracture in Bituminous Materials and Asphaltic Pavements: State-of-the-Art Review
}

Yong-Rak Kim

University of Nebraska-Lincoln, yong-rak.kim@unl.edu

Follow this and additional works at: http://digitalcommons.unl.edu/mechengfacpub

Kim, Yong-Rak, "Cohesive Zone Model to Predict Fracture in Bituminous Materials and Asphaltic Pavements: State-of-the-Art Review" (2011). Mechanical \& Materials Engineering Faculty Publications. 142.

http://digitalcommons.unl.edu/mechengfacpub/142

This Article is brought to you for free and open access by the Mechanical \& Materials Engineering, Department of at DigitalCommons@University of Nebraska - Lincoln. It has been accepted for inclusion in Mechanical \& Materials Engineering Faculty Publications by an authorized administrator of DigitalCommons@University of Nebraska - Lincoln. 


\title{
Cohesive Zone Model to Predict Fracture in Bituminous Materials and Asphaltic Pavements: State-of-the-Art Review
}

\author{
Yong-Rak Kim \\ Department of Civil Engineering, 362M Whittier Research Center, University of Nebraska, Lincoln, \\ NE 68583, USA \\ Corresponding author - Yong-Rag Kim, email: ykim3@unl.edu
}

\begin{abstract}
Cohesive zone (CZ) modeling has been receiving increasing attention from the asphaltic materials and pavement mechanics community as a mechanistic approach to model crack initiation and propagation in materials and structures. The CZ model provides a powerful and efficient tool that can be easily implemented in existing computational methods for brittle, quasi-brittle, and ductile failure as well as interfacial fracture, all of which are frequently observed in asphaltic materials. Accordingly, this paper introduces the $\mathrm{CZ}$ modeling approach in the form of a state-of-the-art review addressing the concept of $\mathrm{CZ}$ modeling, $\mathrm{CZ}$ constitutive relations, their implementation into computational methods, and up-to-date applications of $\mathrm{CZ}$ modeling to bituminous mixtures and pavement structures. This paper also includes a brief discussion on the current challenges that researchers face and the future directions to the modeling of fracture in bituminous materials and pavements. $C Z$ modeling is not a topic that can be possibly discussed in a single article; therefore, it should be clearly noted that this review primarily attempts to deliver some of the core aspects of CZ modeling in the area of bituminous composites.
\end{abstract}

Keywords: cohesive zone model, fracture, bituminous mixtures, asphalt pavement 


\section{Introduction}

As shown in figure 1, various asphalt pavement distresses are related to fracture, including fatigue cracking (both top-down and bottom-up), thermal (transverse) cracking, and reflective cracking, of the asphalt layer. The fracture resistance of asphalt materials significantly influences the service life of asphalt pavements and consequently the maintenance and management of the pavement network.

(a) Bottom-up crack

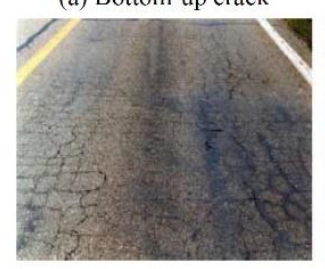

(b) Top-down crack

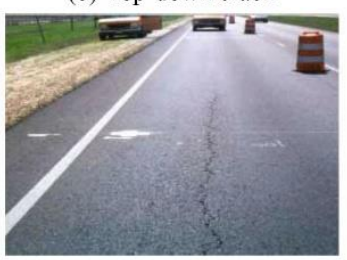

(c) Thermal crack

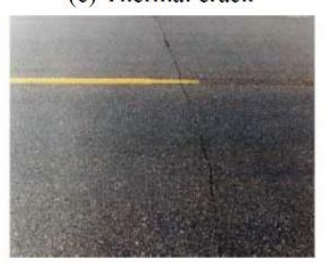

(d) Reflective crack

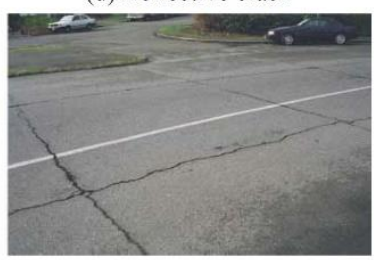

Figure 1. Asphalt pavement distresses related to cracking.

Probably, the most developed and successful approach for modeling damage evolution in bituminous materials is the continuum damage mechanics (CDM) approach, as many researchers (Kim and Little 1990, Park et al. 1996, Lee et al. 2000, 2003, Chehab et al. 2002, 2003, Daniel and Kim 2002, Gibson et al. 2003, Tashman et al. 2004, Chehab and Kim 2005, Masad et al. 2005, Darabi et al. 2011) have studied. More specifically, researchers (Chehab et al. 2002, 2003, Darabi et al. 2011) took into account the major components of asphalt mixture behavior (elastic, viscoelastic, plastic, and viscoplastic) based on a work potential theory, the elastic-viscoelastic correspondence principle, time-temperature superposition with growing damage and a strain-hardening model for viscoplastic behavior to demonstrate good agreement between model predictions and asphalt mixture performance testing results. The CDM approach is relatively simple to apply because it treats the heterogeneous mixtures as macroscopically homogeneous continua.

In spite of significant versatility and simplicity in characterizing structural degradation and damage evolution of bituminous materials, the CDM approach is somewhat limited as it does not account for individual and interrelating effects of mixture constituents and directly measured or measurable physical damage phenomena such as micro-and macrocracks. Various types of damage, including cracks in the asphalt sample, are spatially averaged and represented by stiffness reduction in the asphalt sample as a function of damage parameters. The damage parameters are determined by fitting the model to experimental macroscopic behavior of the sample. Therefore, the CDM approach is limited in capturing and predicting the formation and propagation of a few dominant cracks leading to structural failure. Furthermore, it is limited to address damage behavior related to interactions of small-scale mixture constituents.

One of the most powerful tools for studying fracture processes and damage evolutions of engineering materials is fracture mechanics. Fracture mechanics is the study of the re- 
sponse and failure of structures as a consequence of crack initiation and propagation. Fracture mechanics approaches directly deal with discrete internal boundaries (cracks) in a body, and damage evolution is characterized by employing certain fracture criteria governing the prediction of internal boundaries.

Since Majidzadeh et al. (1971) introduced the fracture mechanics concepts to the field of pavements, the fracture mechanics approach has been widely used in characterizing and predicting pavement cracking analysis. Different from CDM approaches, the fracture mechanics approach can account for the initiation of microcracks and their coalescence to macrocracks, followed by a complete failure of the structures. Therefore, the fracture mechanics approach reflects detailed damage evolution characteristics that are based on the underlying physics of the damage.

Perhaps, the first great development in understanding the mechanics of fracture is by Griffith (1920) who postulated that crack extension will occur in an object when the energy released to produce unit area of crack is equal to or greater than the critical energy release rate of the object, namely $G \geq G$. The critical energy release rate is considered a material constant, which is quite true for some solids including many linear elastic materials. However, traditional linear elastic fracture studies are not valid when the crack tip is subjected to certain level of plastic yielding, which in turn in a case where fracture process zones are large, and the interaction between the process zone and free boundaries can influence the behavior. Consequently, $G_{c}$ is not a material constant, but can depend on specimen size, as intensively discussed in a book (Bazant and Planas 1998). Furthermore, for various viscoelastic media such as bituminous paving mixtures, numerous observations suggest that the critical energy release rate is perhaps better represented as a material parameter not a material constant, because it depends on the loading rate or even the loading history (Knauss 1970, Christensen 1979, Costanzo and Allen 1993, Yoon and Allen 1999). Most traditional fracture mechanics approaches assume the existence of infinitely sharp crack tips leading to stress singularities preceding the crack tips. However, in actual materials (rather than in the conceptual situations), neither the sharpness of the crack nor the stress singularity of the crack tip is often observed; therefore, an alternative approach is necessary for a more appropriate fracture characterization.

As an alternative, the cohesive zone $(\mathrm{CZ})$ modeling approach has received increased attention from the asphaltic materials and pavement mechanics community. It is a wellestablished way to model crack development in monolithic and composite materials not only because it removes the stress singularity at the crack tip but also because it provides a powerful and efficient tool that can be easily implemented in various computational methods, such as finite element and discrete element methods (FEM and DEM). Moreover, the $\mathrm{CZ}$ approach can model both brittle failure and ductile failure, which are frequently observed in asphaltic materials due to the wide range of service temperatures and loading rates. In addition, the $\mathrm{CZ}$ approach can model cracks along bimaterial interfaces (such as aggregate-asphalt interfaces), which are often considered weak zones susceptible to cracking. In comparison, the typical elastic fracture mechanics theories are limited to modeling damage evolution because of crack growth along the bimaterial interfaces.

To that end, this paper introduces the $\mathrm{CZ}$ approach as a useful tool to model crack development in asphaltic materials and pavements. This paper is in the form of a state-of- 
the-art review addressing the concept of $\mathrm{CZ}$ modeling, $\mathrm{CZ}$ constitutive relations and their implementation into computational methods, and offers up-to-date applications of $\mathrm{CZ}$ modeling to bituminous materials and structures. CZ modeling is not a topic that can be possibly discussed in a single article; therefore, it should be clearly noted that this paper primarily attempts to deliver some of the core aspects of $\mathrm{CZ}$ modeling, and applications mostly targeting bituminous composites. This paper also includes a brief discussion on the current challenges that researchers face and future directions to the modeling of fracture in bituminous materials and pavements. It is the author's hope, through this paper, to have readers capture any insights into the $\mathrm{CZ}$ modeling concept, its usefulness and pros and cons in the modeling of fracture-related behavior of bituminous materials and structures, so that a better use of it can be achieved.

\section{Fracture process behavior and cohesive zone modeling}

The fracture process zone is a nonlinear zone characterized by progressive softening, for which the stress decreases at increasing deformation. The nonlinear softening zone is surrounded by a nonsoftening nonlinear zone, which represents material inelasticity. Bazant and Planas (1998) skillfully classified the fracture process behavior in certain materials into three types: brittle, ductile and quasi-brittle. As shown in figure 2 (reproduced from Bazant and Planas 1998), each type presents different relative sizes of those two nonlinear zones (i.e., softening and nonsoftening nonlinear zones) and of the structure.

(a) Brittle

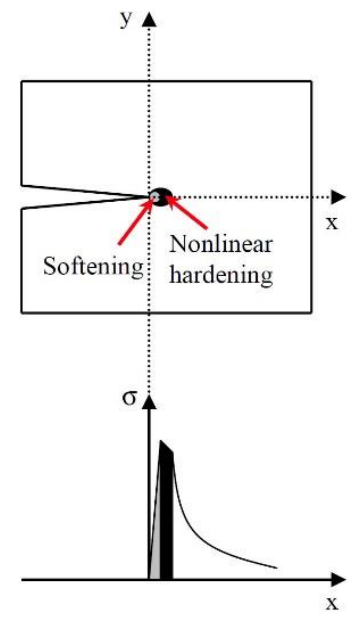

(b) Ductile

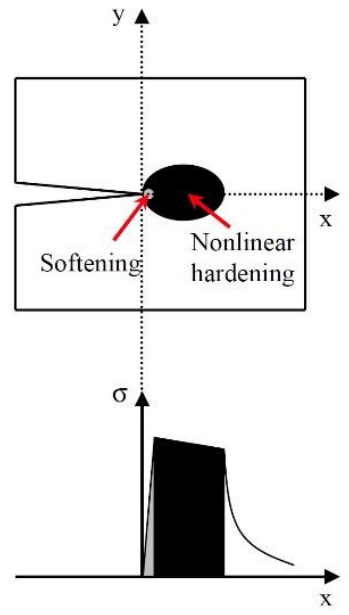

(c) Quasi-brittle

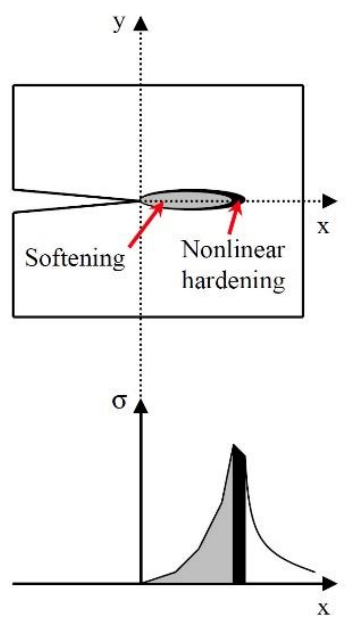

Figure 2. Types of fracture process zone (reproduced from Bazant and Planas 1998).

In the first type of fracture behavior (fig. 2(a)), the whole nonlinear zone is small compared to the structure size, and both the softening zone and nonlinear hardening zone are very small. Then, the entire fracture process takes place almost at the crack tip, and linear elastic fracture mechanics (LEFM) can be successfully used. Brittle materials such as glass, 
brittle ceramics and brittle metals show this type of fracture process behavior. In the second (fig. 2(b)) and third (fig. 2(c)) types of behavior, the ratio of the nonlinear zone size to the structure size is not small; hence, LEFM is inappropriate. In the second type of fracture behavior, as can be seen in the figure, most of the nonlinear zone consists of the inelastic hardening zone (plastic yielding), and the size of actual fracture process (softening) is still small. Many ductile materials (e.g., ductile metals and tough alloys) fall into the second behavior type, and the elasto-plastic fracture mechanics (EPFM) is typically used for this type of fracture behavior. The third type of behavior includes situations in which a major part of the nonlinear zone undergoes progressive damage with material softening due to microcracking, void formation, interface breakages, frictional slips, and others. The softening zone is then surrounded by the inelastic material yielding zone, which is much smaller than the softening zone; therefore, it is often negligible in the analysis. This behavior we call quasi-brittle fracture includes a large fracture process zone that should be taken into account in calculations. Various civil engineering materials such as concrete, rock, coal, cemented sands, stiff clays, woods, and various toughening ceramics present the quasibrittle fracture behavior.

The nonlinear fracture process can be modeled by the $\mathrm{CZ}$ or cohesive crack (Bazant and Planas 1998) model. CZ models regard fracture as a gradual phenomenon in which separation takes place across an extended crack tip, or CZ (fracture process zone), and where fracture is resisted by cohesive tractions. Conceptually, a cohesive crack is a fictitious crack that is able to transfer stress from one face to the other. This model was introduced in the early 1960s by Dugdale (1960) and Barenblatt (1962). Cohesive cracks have since been used by a number of researchers to describe the near-tip nonlinear zone for cracks in most engineering materials: metals, polymers, ceramics, and geomaterials. As one of the noteworthy studies in the area of cementitious materials, Hillerborg et al. (1976) extended the concept of cohesive crack for concrete by proposing that the cohesive crack can develop anywhere in a structure, although there is no preexisting crack. They called this extension the fictitious crack model (Hillerborg et al. 1976, Gustafsson 1985, Hillerborg 1985). The fictitious crack model is mathematically identical to the classical cohesive crack model when the fictitious crack model is used to describe the behavior of a preexisting crack (Bazant and Planas 1998).

Park (2009) skillfully introduced the nonlinear fracture process of the CZ model with four stages as shown in figure 3. The first stage (Stage I) represents general material behavior without damage. The next stage (Stage II) is the initiation of a crack when a certain criterion such as a maximum stress $\left(\sigma_{\max }\right)$ shown in the figure is met. The third stage (Stage III) describes the nonlinear material softening that characterizes damage evolution, and the final stage (Stage IV) defines failure with a critical crack opening width $(\delta)$ representing the new surfaces created by the fracture process, which have no traction (no loadbearing capacity). Simply put, with increasing separation $(\Delta)$ the traction $(T)$ increases, reaches a maximum (cohesive strength $\sigma_{\max }$ ), and then, governed by the softening curve ( $T$ as a function of $\Delta$ ), decreases and eventually vanishes at the critical separation ( $\delta$ ) creating tractionfree crack surfaces. The $\mathrm{CZ}$ surface sustains a distribution of tractions that are a function of the displacement jump across the surface. The relationship between the $\mathrm{CZ}$ tractions 
and the separation displacements (or equivalently crack opening widths) identifies the shape of the softening curve (Stage III) and defines the constitutive law for the CZ.

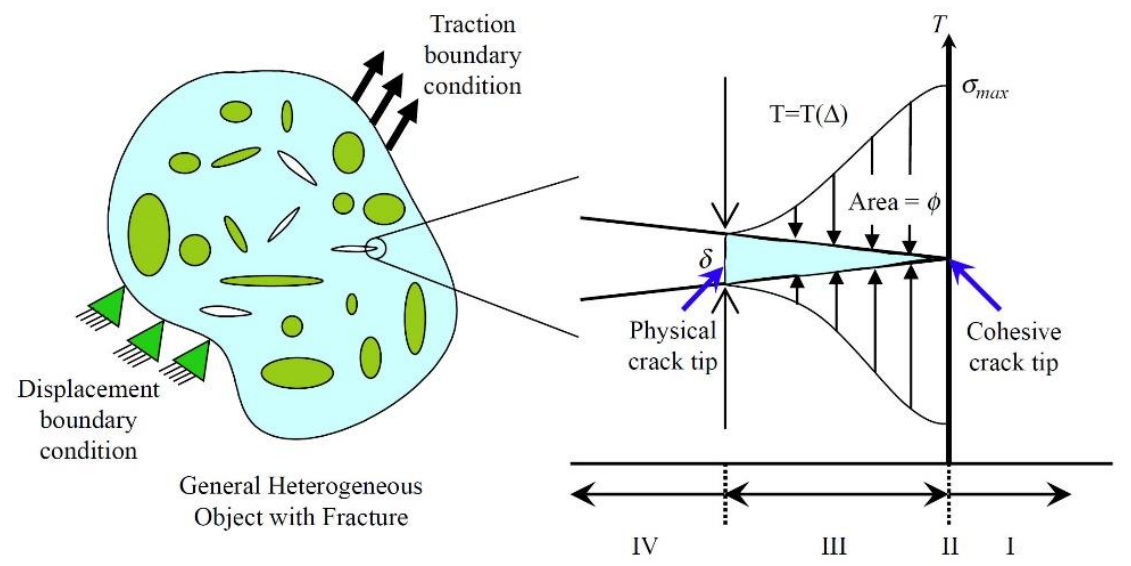

Figure 3. Schematic illustration of the cohesive zone model.

\section{Cohesive zone constitutive relations}

An important issue on the use of $\mathrm{CZ}$ model is the determination of the relationship between the $\mathrm{CZ}$ tractions and the separation displacements. In particular, the relevant fracture parameters such as cohesive strength $\left(\sigma_{\max }\right)$, critical separation $(\delta)$, and fracture energy $(\phi)$ as represented in figure 3 , as well as the shape of the softening curve (traction-separation curve), must be identified. A number of different $\mathrm{CZ}$ models have been developed and proposed by researchers as presented in many literatures (Dugdale 1960, Barenblatt 1962, Needleman 1987, 1990, Rice and Wang 1989, Tvergaard 1990, Tvergaard and Hutchinson 1992, 1993, Xu and Needleman 1993, Allen et al. 1994, Camacho and Ortiz 1996, Geubelle and Baylor 1998, Ortiz and Pandolfi 1999, Yoon and Allen 1999, Allen and Searcy 2001, Yang and Thouless 2001, Espinosa and Zavattieri 2003, Zhang and Paulino 2005, Shim et al. 2006, Park et al. 2009). Depending on the prefracture response of the cohesive surfaces, each model can be classified by either intrinsic or extrinsic. The prefracture response is characterized by the existence of an artificial initial stiffness in the CZ model. Models for which the artificial initial stiffness is considered are called intrinsic models, whereas models assuming initial rigidity for the CZs are called extrinsic models.

In the intrinsic models, as illustrated in figure $4(a)$, the traction-separation relation is such that with increasing separation, the traction across the $\mathrm{CZ}$ reaches a maximum, then decreases and eventually vanishes, indicating a complete decohesion (separation). In contrast, the extrinsic CZ model, as shown in figure 4(b), does not display the initial ascending trend in the traction-separation curve. It is assumed that separation occurs when $\mathrm{CZ}$ traction reaches the cohesive strength of the material, and once the separation occurs, the $\mathrm{CZ}$ traction decreases as separation continues. 
(a)

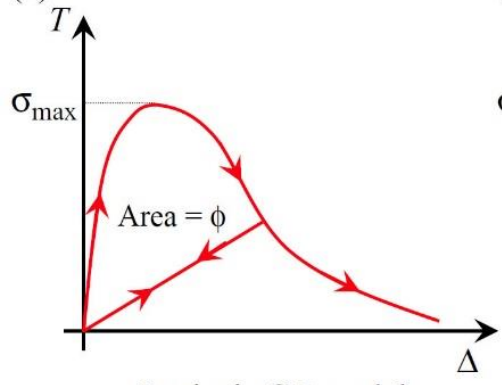

Intrinsic $\mathrm{CZ}$ model (b)

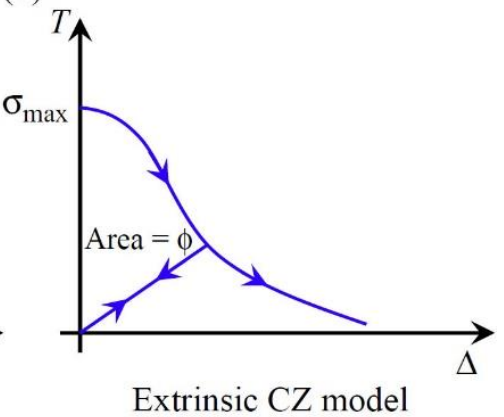

Figure 4. CZ traction-separation relations: (a) intrinsic model and (b) extrinsic model.

Intrinsic formulations are easier to implement but may become very computationally expensive because many cohesive elements, some of which are not really necessary, need to be inserted a priori within the finite element meshes. On the other hand, the extrinsic models are probably more realistic than the intrinsic approach, because they do not assume the preexistence of $\mathrm{CZ}$ elements within the finite element meshes. This characteristic is generally referred to as "initial rigidity," as illustrated in figure 4(b).

Shet and Chandra (2002) summarize some of the popular CZ models developed by researchers with specific purposes regarding the shape of the $\mathrm{CZ}$ traction-separation curve and the model parameters involved. Representative problems attempted and solved using each CZ model are also presented in the study. The work of separation, cohesive strength and critical displacement is typically the key parameters that characterize individual $\mathrm{CZ}$ models. As presented in the study, the $\mathrm{CZ}$ model has been used in various applications and materials (ductile, quasi-brittle, and brittle) with and without rate-dependent mechanical behavior, which infers that it can provide an ideal framework to model strength, stiffness, and failure in an integrated manner.

\section{Computational implementation of cohesive zone models}

Perhaps, the success and popularity of the $\mathrm{CZ}$ modeling of fracture are because it provides a powerful and efficient tool that can be easily implemented in various computational methods such as FEM and DEM. Regardless of the origin in developing the CZ model (i.e., potential-based or non-potential-based), equations relating normal and tangential displacement jumps across the cohesive surfaces with proper tractions define a CZ model, and the $\mathrm{CZ}$ model is implemented numerically via interface elements.

A CZ element in the finite element mesh consists of two line elements (i.e., cohesive surfaces) that connect the faces of adjacent elements during the fracture process. The two lines (surfaces) initially lie together as the intact state but are progressively separated as the level of damage at the $\mathrm{CZ}$ increases. The damage evolution followed by a complete separation is controlled by the corresponding traction-separation relationship.

In an attempt to illustrate differences between the two $\mathrm{CZ}$ implementation techniques (i.e., intrinsic and extrinsic), figure 5 is introduced herein. Figure 5 represents a typical two- 
phase composite in which black rigid particles are embedded in white matrix. As shown in figure 5(a), the two-phase composite is subjected to uniaxial tensile loading condition by imposing the following boundary conditions: vertical and horizontal displacements at the bottom face are constrained, and uniaxial tensile displacements are applied to the top face at a constant rate $\left(U_{\mathrm{o}}\right)$.

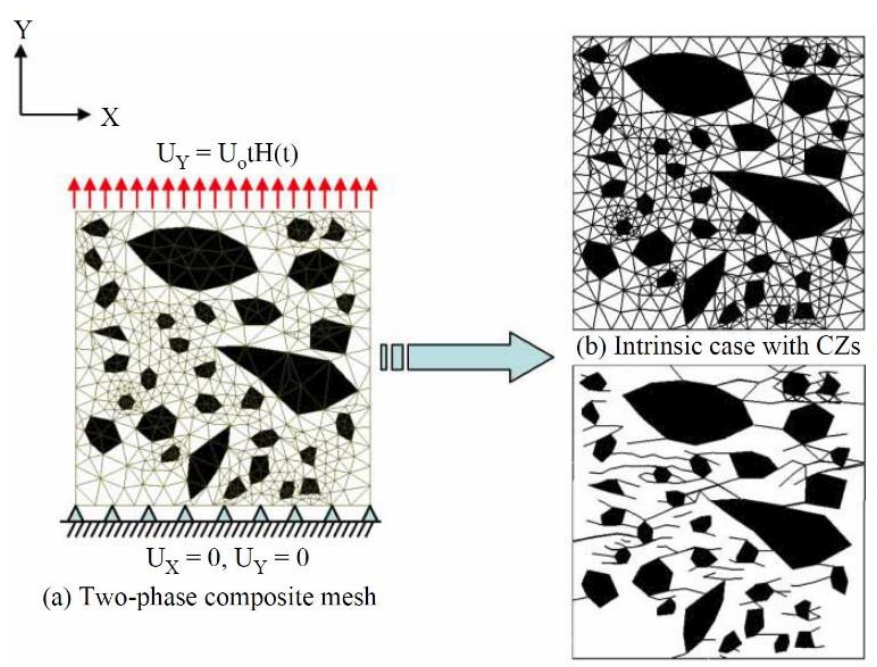

(c) Extrinsic case with CZs

Figure 5. Finite element mesh of a two-phase composite and its CZ developments simulated by two different implementation techniques: intrinsic vs. extrinsic.

Assuming CZ elements signifying damage evolution due to crack growth are only placed within the white matrix phase and are not developed within rigid black particles, finite element simulation results from the two different modeling approaches (intrinsic vs. extrinsic) are shown in figure 5(b), (c), respectively, in the form of snapshots taken at the same loading level. Dark black lines within the white matrix phase represent CZ elements involved in the damage process.

As shown in figure 5(b), in the case of intrinsic modeling, the possible cracked configurations of the bodies are governed by the topology of the finite element discretization, which implies mesh-dependent simulation results. Finite element simulation results are affected by the size and orientations of $\mathrm{CZ}$ elements, which is due to the fact that the $\mathrm{CZ}$ is represented by a highly nonlinear relation between the traction and displacement jump. In order to capture the temporal- and spatial-dependent development of cracks properly, enough number of $\mathrm{CZ}$ elements needs to be placed in an optimized manner, otherwise considerable accuracy can be lost. This mesh dependence has been reported by many researchers (Xu and Needleman 1994, Miller et al. 1999, Klein et al. 2001, Scheider and Brocks 2003).

Several researchers have also reported numerical issues associated with artificial compliance in the response of the bodies of interest to externally applied loads due to the assumption of the initial artificial stiffness in the traction-separation relations of intrinsic $\mathrm{CZ}$ 
models. This phenomenon is related to the assumption made in intrinsic $\mathrm{CZ}$ models that discontinuities in the displacement fields happen in the uncracked body. As a result, an artificial elasticity rises at the inter-element boundaries and the corresponding stiffness of that imaginary spring introduced between bulk elements depends on the initial stiffness of the intrinsic CZ model. The artificial compliance effect can be significant and results in an adverse numerical impact when $\mathrm{CZ}$ elements are embedded in a large area in the object and/or when the initial ascending slope is not sufficiently steep. This problem may be effectively controlled by specifying very high values of initial stiffness in the CZ model (Geubelle and Baylor 1998, Espinosa et al. 2000, Alfano and Crisfield 2001, Klein et al. 2001, Zavattieri and Espinosa 2001, Espinosa and Zavattieri 2003, Song et al. 2006a). However, the use of "too stiff" initial cohesive stiffness can generate problems with stability and severely restrict the simulation time steps. Espinosa and Zavattieri (2003) suggested a scheme in which two time steps, the standard time step related to the properties of the bulk material and the second time step named by the authors as the cohesive time step, are calculated and the smallest between the two is taken as the overall time step.

In the case of extrinsic modeling, as clearly demonstrated in figure 5(c), cohesive elements are inserted as needed by node duplication (the process generally referred to as dynamic or adaptive insertion) in the mesh whenever a damage initiation criterion is reached. This initial rigid response lets the adjacent continuum elements remain concurrent before initiation of the fracture process, eliminating the problem of artificial compliance prior to fracture. The node duplication process is followed by gradual reduction in the load-bearing capacity of the cohesive element and the eventual complete failure of that element after the characteristic interfacial separation is reached. However, this adaptive insertion scheme requires extra implementation efforts because it requires the continuous update of the node numbering within the mesh as edges of continuum elements are duplicated to simulate the propagation of cracks. This topic has been investigated by several researchers (Pandolfi and Ortiz 1998, 2002, Mota et al. 2008, Paulino et al. 2008). Challenges associated with the use of extrinsic $\mathrm{CZ}$ models include the difficulties related to the continuous update of the node numbering as cohesive elements are inserted in the meshes and mesh dependency (Ruiz et al. 2001, Papoulia et al. 2003, Zhou and Molinari 2004). Other researchers have reported problems with what they called time discontinuity of the inter-element tractions (Papoulia et al. 2003, Sam et al. 2005).

In addition, as similar to the intrinsic case, the possible crack development in the body is also affected by the initial configuration of volume elements in the finite element mesh. Therefore, simulation results are dependent on the finite element discretization. For an appropriate level of model accuracy, the mesh needs to be fine enough so as to properly capture the highly nonlinear traction-displacement relation.

\section{Application of cohesive zone model to asphalt mixtures and pavements}

The CZ modeling approach in asphalt materials and flexible pavements was probably first employed by Jeng and his colleagues (Jeng and Perng 1991, Jeng et al. 1993) to model crack resistance and propagation in asphalt pavement overlays. Over the past several decades, two primary attempts have been made by various researchers for the use of the $\mathrm{CZ}$ concept 
to the analysis and modeling of fracture process in asphaltic materials and pavements. One of the primary efforts has been pursued to estimate fracture properties (i.e., $\mathrm{CZ}$ parameters) of the materials so that the properties can be used to predict fracture-related damage evolution in the mixtures and structures. For a more appropriate estimation, researchers have sometimes modified preexisting fracture test methods or even newly designed their own testing protocols because preexisting fracture test methods could not properly capture fracture processes of highly nonlinear, inelastic, and heterogeneous materials such as asphaltic materials. Another challenge that researchers have faced is the use of $\mathrm{CZ}$ models that are tailored for a more accurate estimation of fracture mechanisms and a better performance prediction of fracture-related damage and failure in the asphalt mixtures and pavement structures. This is an extremely complicated issue, because the asphaltic mixtures and structures are highly heterogeneous, inelastic, nonlinear, rate dependent, and temperature sensitive, and demonstrate cracks in multiple length scales incorporated with multiphysical aspects such as moisture damage and material aging. To that end, this section introduces some recent attempts (obviously not all) made by civil engineering researchers to advance fracture test methods that characterize the $\mathrm{CZ}$ fracture parameters and to explore $\mathrm{CZ}$ models that are more accurately represent the fracture process of bituminous materials in a summarized form.

\section{Experimental efforts to characterize the cohesive zone fracture properties}

To characterize the fracture properties of asphalt mixtures, three geometries have typically been pursued by researchers. They are (1) single-edge notched beam (SE(B)) specimen, (2) disc-shaped compact tension (DC(T)) specimen, and (3) semicircular bending (SCB) specimen, as shown in figure 6.

(a) $\mathrm{SE}(\mathrm{B})$ Test

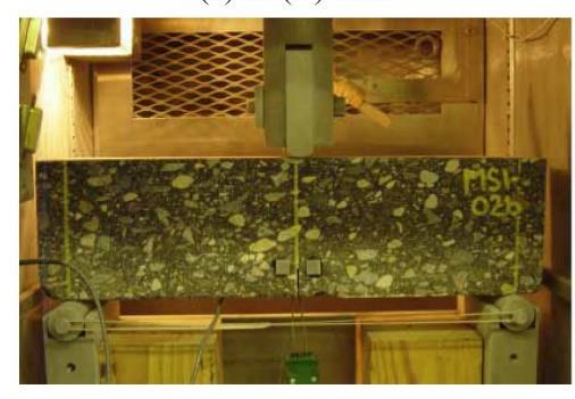

(b) DC(T) Test

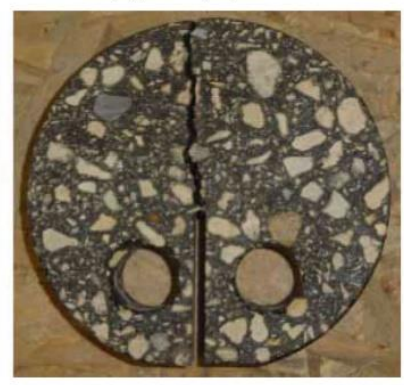

(c) SCB Test

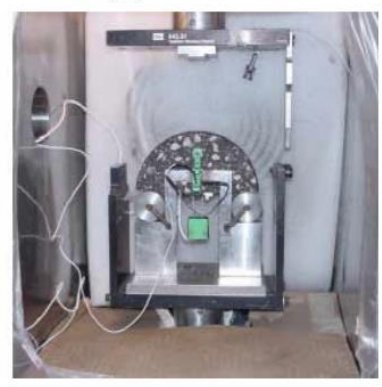

Figure 6. Three primary test methods used to assess asphalt mixture fracture properties.

The most common geometry used for the fracture testing of asphalt concrete has probably been the single-edge notched specimen (SE(B); Mobasher et al. 1997, Marasteanu et al. 2002). To obtain the cohesive fracture energy, Wagoner et al. (2005a) proposed a testing protocol that uses the SE(B) geometry. Wagoner et al. (2005a) concluded in their studies that $\mathrm{SE}(\mathrm{B})$ testing is probably the most promising fracture test based on test control, crack front development, test repeatability, and mixed-mode fracture. Song et al. (2006b) and 
Kim et al. (2009) used the methodology proposed by Wagoner et al. (2005a) to calibrate cohesive fracture parameters used in their FEM and DEM simulations, respectively. The main problems with the use of $\mathrm{SE}(\mathrm{B})$ geometry to routinely obtain fracture properties of asphalt mixtures are that the fabrication of specimens in the laboratory becomes impractical, and that it is not often viable to extract beam specimens from mixtures in the field.

The compact tension test is a part of ASTM E399-90 (2002) and recommended as an alternative to the $\mathrm{SE}(\mathrm{B})$ test. Although the compact tension test can be performed with both rectangular and disc-shaped compact specimens, the $\mathrm{DC}(\mathrm{T})$ specimen has typically been used in the characterization of asphalt concrete fracture properties because the specimen can be easily prepared by compacting asphalt mixtures with a Superpave Gyratory Compactor. Significant work has been conducted by researchers (Wagoner et al. 2005b, 2005c) on the development of the DC(T) fracture testing protocol. They modified the original ASTM DC(T) geometry, which is usually for metallic materials, by moving the location of the loading holes to reduce failure at the loading holes. The researchers also set the thickness of the specimens to $50 \mathrm{~mm}$ and the notch length to $19 \mathrm{~mm}$ to maximize the ligament area. At different testing temperatures $\left(-20,-10\right.$ and $\left.0^{\circ} \mathrm{C}\right)$ and loading rates, the fracture energy was simply computed by the area under the load-crack mouth opening displacement curve normalized by the area of the fracture surface. The researchers found that as the temperature increased, the fracture energy increased, and as the loading rate increased, the fracture energy decreased. The DC(T) test is now specified as ASTM D7313-07a (2007).

One simpler alternative to the $\mathrm{SE}(\mathrm{B})$ and the $\mathrm{DC}(\mathrm{T})$ is the $\mathrm{SCB}$ test originally proposed by Chong and Kuruppu $(1984,1988)$. The SCB specimen has since been used by many researchers (Chong et al. 1989, Basham et al. 1990, Khalid and Artamendi 2008, Mohammad and Kabir 2008, van Rooijen and de Bondt 2008) to obtain the fracture toughness, fracture energy and stress-softening curves of various types of brittle and quasi-brittle materials. The SCB is advantageous due to its relatively simple testing configuration, more economical aspects in specimen fabrication (two testing specimens are produced from one cylinder sample) and repeatable testing results. Another benefit of SCB testing is that it can possibly be used to determine mixed-mode (Modes I and II) fracture toughness by adjusting the angle of the notch (Chong and Kuruppu 1988). According to studies (Molenaar and Molenaar 2000, Li 2005, Li et al. 2005), the SCB test can identify fracture characteristics in a sensitive manner depending on the testing temperatures, materials used in the mixtures and loading conditions (e.g., rates). Li (2005) used acoustic emission (AE) techniques incorporated with SCB fracture testing to obtain relevant information about the fracture process in asphalt mixtures. AE is regarded as a useful tool for obtaining information about the microscopic damage during fracture and allows for a better understanding of the relationship between the microstructural events and the macroscopic performance. Although the SCB test geometry has presented advantageous aspects compared to other geometries, several drawbacks should be noticed. Wagoner et al. (2005b) indicated that the $150 \mathrm{~mm}$ diameter of SCB specimens could lead to a relatively short (or insufficient) fracture ligament, which should be as large as possible to produce a reliable fracture property. In addition, the crack propagation with the SCB geometry creates an arching effect with high compressive stress, which further reduces the effective ligament, creating potentially invalid testing results. 


\section{Modeling efforts to address fracture in asphalt mixtures and pavements}

Recently, the CZ modeling concept has been actively implemented in the modeling of asphalt concrete, particularly to simulate fracture with the consideration of the bulk material inelasticity. It is well known that asphaltic materials are rate dependent and temperature sensitive due to the asphalt binder phase in the mixture. Thus, the assumption of linear elasticity for the bulk material is not appropriate to produce accurate results. Examples of research efforts considering the viscoelasticity of the bulk material in asphalt mixtures are presented in various studies including Souza et al. (2004), Kim et al. (2005, 2006, 2007), Song et al. (2006a), Kim and Buttlar (2009) and Aragão et al. (2011). More particularly, Kim et al. $(2005,2006,2007)$ have employed the nonlinear viscoelastic CZ model developed by Allen and his colleagues (Yoon and Allen 1999, Allen and Searcy 2001) to simulate the fracture and damage behavior of asphalt mixtures at intermediate temperatures. In order to predict material-specific and rate-dependent viscoelastic fracture of asphalt media, they performed laboratory tests to obtain the linear viscoelastic properties as well as the nonlinear viscoelastic fracture parameters of the asphalt phase and incorporated those parameters into the FEM model.

Kim et al. (2006, 2007, 2010a, Aragão et al. 2011) have also incorporated the CZ models into a computational modeling framework that considers mixture heterogeneity. As exemplified in figure 7, the approach is based on the finite element modeling of mixture microstructure. Individual mixture components (i.e., asphalt matrix phase, which is subject to cracking, and coarse aggregates that are not associated with fracture) are separately modeled with their material properties measured from laboratory tests.

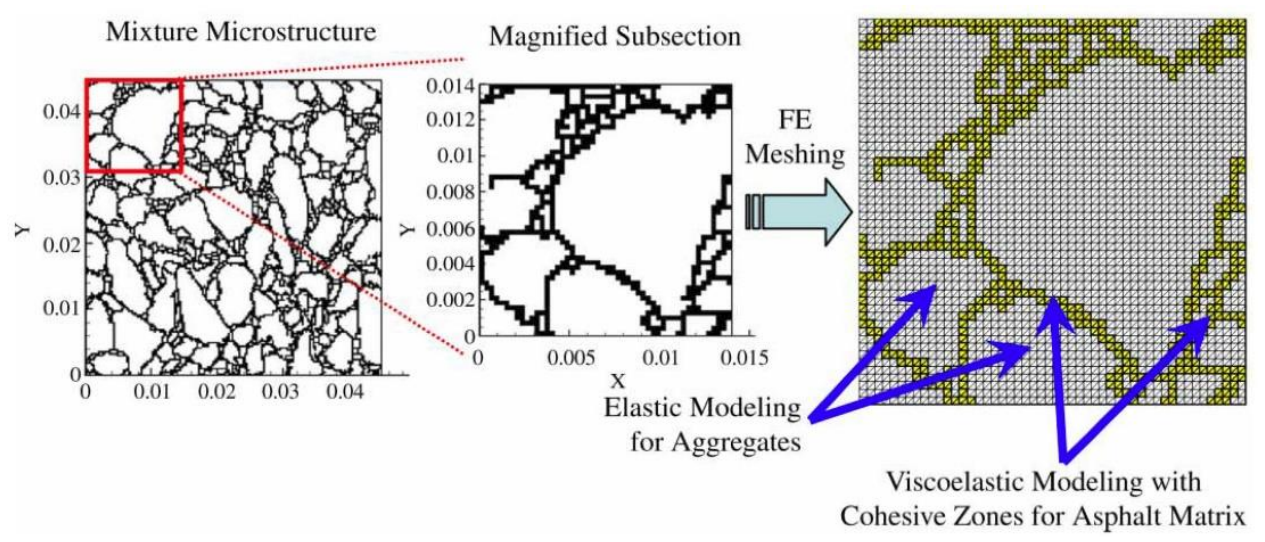

Figure 7. Microstructure finite element modeling with intrinsic cohesive zone elements.

In order to characterize the $\mathrm{CZ}$ fracture properties of the asphalt matrix phase, which is a mixture of asphalt binder and fine aggregates, Aragão and Kim (2010, 2011) have conducted fracture tests of the asphalt matrix mixture with SCB geometry, as shown in figure 8. The testing has been incorporated with the use of a digital image correlation (DIC) sys- 
tem that can monitor the displacement and strain fields on the overall surface of the specimen. The DIC analysis helps characterize the fracture behavior more realistically so that the determination of $\mathrm{CZ}$ properties can be more effectively performed.

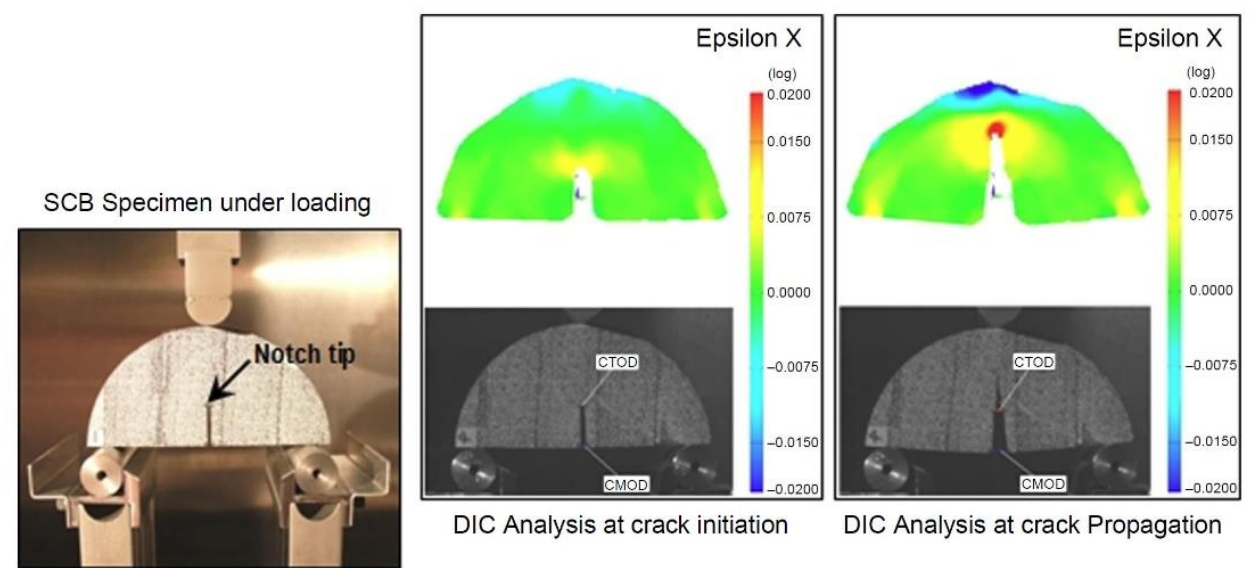

Figure 8. SCB fracture test of an asphalt matrix sample incorporated with the DIC.

The computational approach considering mixture heterogeneity has been attempted by many researchers (Buttlar and You 2001, Masad et al. 2001, Papagiannakis et al. 2002, Sadd et al. 2003, Soares et al. 2003, You and Buttlar 2004, 2005, 2006, Abbas et al. 2005, Dai et al. 2005, Dai and You 2007, Kim and Lutif 2008, You et al. 2009, Aragão et al. 2010) because it can effectively account for multiple length scales and mixture heterogeneity by considering individual mixture constituents separately with the aid of well-developed numerical techniques. The computational modeling approach incorporated with the mixture microstructure and CZ fracture can produce great benefits in the sense that this approach requires only the fundamental properties of mixture constituents for the simulation, which results in considerable savings in both time and cost because the model can significantly reduce time-consuming and expensive laboratory performance tests of mixture samples.

Although the computational techniques based on the mixture microstructure have been shown to be extremely versatile in directly addressing the effects of heterogeneity, inelasticity and damage growth in multiple forms in the asphaltic mixtures and structures, the asphaltic composites that contain thousands of irregularly shaped, randomly oriented inclusions (particles, voids, etc.) along with a number of potential crack sites at different length scales would require a highly refined mesh. The solution for such problems requires the use of a tremendous amount of computational time and effort, which is not always feasible with the currently available computing power. These limitations have led researchers to seek alternative approximate approaches that can account for the hierarchical structure of heterogeneous materials without having to model every microstructural detail, but still considering the most important ones.

To that end, researchers have developed and used a multiscale modeling approach (Feng et al. 2008, Soares et al. 2008, 2009, Kim et al. 2010a, 2010b, Lutif et al. 2010), which 
has rarely been applied to pavement materials and structures, as an ideal alternative to predict larger scale structural behavior with much less computational effort but still considering microstructure details and fracture in multiple length scales. The multiscale modeling has been attempted by many researchers in various disciplines, as demonstrated in a number of studies (Fish and Wagiman 1993, Fish and Belsky 1995, Oden and Zohdi 1997, Feyel 1999, Lee et al. 1999, Oden et al. 1999, Feyel and Chaboche 2000, Fish and Shek 2000, Ghosh et al. 2001, Raghavan et al. 2001, Haj-Ali and Muliana 2004, Souza and Allen 2010). In the multiscale approach, a separate scale analysis is performed at each of the smaller structural scales within the macroscopic body. Damage can also be modeled explicitly at each length scale by incorporating appropriate types of fracture/damage mechanics modeling such as the $\mathrm{CZ}$ model into the analysis.

Another interesting implementation of the $\mathrm{CZ}$ concept in the damage modeling of asphaltic materials has been pursued by Caro et al. (2010a, 2010b). They used a commercial finite element package, ABAQUS, to simulate moisture-induced damage in asphalt concrete mixtures. Based on the sequentially coupled modeling technique, they studied the effect of moisture diffusion with the mechanical performance of asphalt mixtures by means of two different mechanisms: reduction in the linear viscoelastic relaxation modulus of the asphalt matrix as a function of the amount of moisture present in the material and deterioration of the adhesive fracture properties of the asphalt matrix-aggregate interfaces as a function of the amount of moisture. They simulated the nucleation, initiation and propagation of adhesive fracture at the aggregate-asphalt matrix interfaces through the CZ modeling technique.

The CZ model has also been applied to the fracture simulations of pavement structures. Baek and Al-Qadi (2008) used 3D finite element-based CZ model to investigate the reflective cracking mechanisms in hotmix asphalt (HMA) overlays and the ability of the steel reinforcement netting interlayer to retard reflective cracking. The study found that steel reinforcement netting placed between the HMA overlay and existing cement concrete pavement significantly delays reflective crack initiation. Another recent study was performed by Kim and Buttlar (2009), who employed the CZ model implemented in the FEM to investigate the low-temperature fracture behavior of airport pavements. The authors demonstrated that the finite element pavement fracture models could successfully predict the progressive crack behavior of asphalt pavements under critical temperature and heavy aircraft gear loading conditions.

As clearly indicated from the numerous efforts introduced herein, the $\mathrm{CZ}$ approach can be used to model various fracture-related events in many different asphaltic materials, mixtures and structures because of its excellent flexibility and compatibility with the commonly used numerical techniques: FEM and DEM.

\section{Current challenges and future directions}

In spite of the great promises and potential of the CZ-based fracture approaches, a number of challenges need to be overcome in order to move them into real practice. There is no test method that can comprehensively define the fracture properties of asphalt materials sub- 
jected to various environmental conditions (temperatures and moisture) and loading conditions. At the current stage, no fracture test is available to accurately address mixed-mode fracture behavior, which is typically observed in pavement fracture. Most of the fracture test methods have mainly focused on identifying Mode I (opening-mode) fracture. In addition, no fracture model has been fully validated yet. The model for the next generation should appropriately address the current modeling challenges such as nonlinear-inelastic material behavior including the rate-dependent fracture, mixture heterogeneity, microstructure-dependent characteristics and the multiscale-multiphysics mechanical phenomenon. In these endeavors, numerical approaches incorporated with the CZ model seem to be appropriate because they can account for the different properties of the various mixture constituents, the heterogeneity of the mixtures, and the fracture process on a more realistic scale. However, as noted in many recent studies aforementioned in this paper, computational microstructure modeling has shown many technical challenges including (1) the proper consideration of air voids within the mixtures, (2) consideration of material-tomaterial interactions and (3) intensive computations (poor computational efficiency). The computational efficiency is greatly intensified if three-dimensional simulations are pursued.

Although a lot of problems and issues are raised, given the continuous growth expected in the computational power for the next decades and the various advanced ideas such as multiscale modeling, the extended FEM (the so-called XFEM or similarly the generalized FEM, G-FEM) proposed by researchers in other engineering/physics disciplines (Duarte and Oden 1996, Babuska and Melenk 1997, Daux et al. 2000, Duarte et al. 2000,Wells and Sluys 2001, Moes and Belytschko 2002, Belytschko et al. 2003), the mechanistic approaches to model fracture and damage in bituminous mixtures and pavements seem appropriate and unavoidable. These can provide powerful tools to accurately predict the mechanical responses of the complicated asphalt materials and mixtures with a maximized efficiency.

In preparation for the coming decades, efforts toward CZ modeling incorporated with microstructure characterization and multiscale and multiphysics modeling are timely and necessary; they reflect contemporary understanding of the analysis modeling approach of bituminous mixtures - a clear shift from the use of empirical approaches to the use of fundamental mechanics and materials science considering hierarchical length scales and microstructure. The success of the next generation performance prediction depends on the availability and quality of the model.

\section{Concluding remarks}

In the past several decades, bituminous materials/pavement researchers have performed extensive studies to better understand the mechanisms of various forms of cracking and to develop new test procedures and prediction models to improve asphalt pavement performance. One of the most notable trends is that more and more researchers rely on fracture tests based on the fundamentals to evaluate cracking resistance in a more accurate manner. Clearly, significant advances have been made over the past decade in the development of asphalt material fracture tests such as the SCB test and the DC(T). These tests have been 
found to be vastly superior in their ability to distinguish between mixtures with different mixture ingredients.

Along with the use of fundamental fracture tests, the community has been interested in the use or development of better cracking models. Empirical models are typically limited to explain the cracking phenomenon at a fundamental level. Conversely, mechanics-based models rely on advanced mechanistic theories in describing the cracking process; therefore, these models can provide predictions that are more accurate and are not limited to specific cases. The mechanistic cracking models are generally considered more complex and hard to implement, but the $\mathrm{CZ}$ modeling approach is relatively clear to understand and provides vast benefits to the implementation with typical numerical techniques such as the FEM. Furthermore, the CZ models directly utilize laboratory fracture test data. These tools represent the cutting edge and, if deemed useful, could be feasibly implemented as a replacement for the fracture "engine" in the pavement design guide.

Application of the $\mathrm{CZ}$ modeling approaches to practical problems in our community does not seem to occur in the very near future at this moment. A lot is still to be learned, and the approach remains many challenges and questions. Nevertheless, efforts toward the mechanistic fracture approach, such as the $\mathrm{CZ}$ modeling, are timely and necessary for the future due to their significant benefits and potential. They reflect the contemporary shift from the empirical approaches to the use of mechanics and fundamentals in the analysis and modeling of bituminous mixtures and asphaltic pavements.

\section{References}

Abbas, A., et al., 2005. Modeling of asphalt mastic stiffness using discrete elements and micromechanics analysis. International Journal of Pavement Engineering, 6 (2), 137-146.

Alfano, G., and Crisfield, M. A., 2001. Finite element interface models for the delamination analysis of laminated composites: mechanical and computational issues. International Journal for Numerical Method in Engineering, 50, 1701-1736.

Allen, D. H., Jones, R. H., and Boyd, J. G., 1994. Micromechanical analysis of a continuous fiber metal matrix composite including the effects of matrix viscoplasticity and evolving damage. Journal of Mechanics and Physics of Solids, 42 (3), 505-529.

Allen, D. H., and Searcy, C. R., 2001. A micromechanical model for a viscoelastic cohesive zone. International Journal of Fracture, 107, 159-176.

Aragão, F. T. S., and Kim, Y., 2010. Modeling fracture and failure of heterogeneous and inelastic asphaltic materials using the cohesive zone concept and the finite element method, West Palm Beach, FL: CD Publication, 2662-2671.

Aragão, F. T. S., and Kim, Y., 2011. Characterization of fracture properties of asphalt mixtures based on cohesive zone modeling and digital image correlation technique. Washington, DC: Transportation Research Board Compendium CD.

Aragão, F. T. S., et al., 2010. Semi-empirical, analytical, and computational predictions of dynamic modulus of asphalt concrete mixtures. Transportation Research Record, 2181, 19-27.

Aragão, F. T. S., et al., 2011. A micromechanical model for heterogeneous asphalt concrete mixtures subjected to fracture failure. Journal of Materials in Civil Engineering, 23 (1), 30-38.

ASTM E399-90, 2002. Plane-strain fracture toughness for metallic materials. Annual book of ASTM standards. Vol. 03.01. Philadelphia, PA: ASTM. 
ASTM D7313-07a, 2007. Standard test method for determining fracture energy of asphalt-aggregate mixtures using the disk-shaped compact tension geometry. Annual book of ASTM standards. Vol. 04.03. Philadelphia, PA: ASTM.

Babuska, I., and Melenk, J. M., 1997. Partition of unity method. International Journal for Numerical Methods in Engineering, 40 (4), 727-758.

Baek, J., and Al-Qadi, I. L., 2008. Mechanism of overlay reinforcement to retard reflective cracking under moving vehicular loading. Proceedings of the sixth RILEM international conference on cracking in pavements. Chicago, IL: CRC Press, 563-573.

Barenblatt, G. I., 1962. Mathematical theory of equilibrium cracks in brittle fracture. Advances in Applied Mechanics, 7, 55-129.

Basham, K. D., Chong, K. P., and Boseri, A. P., 1990. A new development in devising tension-softening curves for brittle materials. Proceedings of the ninth international conference on experimental mechanics. Copenhagen, Denmark: Technical University of Denmark, 1423-1432.

Bazant, Z. P., and Planas, J., 1998. Fracture and size effect in concrete and other quasi-brittle materials. Boca Raton, FL: CRC Press.

Belytschko, T., et al., 2003. Dynamic crack propagation based on loss of hyperbolicity and a new discontinuous enrichment. International Journal for Numerical Methods in Engineering, 58 (12), 18731905.

Buttlar, W., and You, Z., 2001. Discrete element modeling of asphalt concrete: microfabric approach. Transportation Research Record, 1757, 111-118.

Camacho, G. T., and Ortiz, M., 1996. Computational modelling of impact damage in brittle materials. International Journal of Solids and Structures, 33 (20-22), 2899-2938.

Caro, S., et al., 2010a. Micromechanical modeling of the influence of material properties on moistureinduced damage in asphalt mixtures. Construction and Building Materials, 24 (7), 1184-1192.

Caro, S., et al., 2010b. Coupled micromechanical model of moisture-induced damage in asphalt mixtures. Journal of Materials in Civil Engineering, 22 (4), 380-388.

Chehab, G. R., et al., 2002. Time-temperature superposition principle for asphalt concrete mixtures with growing damage in tension state. Journal of the Association of Asphalt Paving Technologists, 71, 559-593.

Chehab, G., et al., 2003. Characterization of asphalt concrete in uniaxial tension using a viscoelastoplastic continuum damage model. Journal of the Association of Asphalt Paving Technologists, 72, 315355.

Chehab, G. R., and Kim, Y. R., 2005. Viscoelastoplastic continuum damage model application to thermal cracking of asphalt concrete. Journal of Materials in Civil Engineering, 17 (4), 384-392.

Chong, K. P., Basham, K. D. and Wang, D. Q., 1989. Fracture parameters derived from tensionsoftening measurement using semi-circular specimens. In: H. Mihashi, H. Takahashi, and F. H. Wittmann, eds. Fracture toughness and fracture energy. Balkema: Rotterdam, 278-286.

Chong, K. P., and Kuruppu, M. D., 1984. New specimen for fracture toughness determination for rock and other materials. International Journal of Fracture, 26, R59-R62.

Chong, K. P., and Kuruppu, M. D., 1988. New specimens for mixed mode fracture investigations of geomaterials. Engineering Fracture Mechanics, 30 (5), 701-712.

Christensen, R. M., 1979. A rate-dependent criterion for crack growth. International Journal of Fracture, $15(1), 3-21$.

Costanzo, F., and Allen, D. H., 1993. A continuum mechanics approach to some problems in subcritical crack propagation. International Journal of Fracture, 63 (1), 27-57. 
Dai, Q., et al., 2005. Prediction of damage behaviors in asphalt materials using a micromechanical finite-element model and image analysis. Journal of Engineering Mechanics, 131 (7), 668-677.

Dai, Q., and You, Z., 2007. Micromechanical finite element framework for predicting viscoelastic properties of asphalt mixtures. Materials and Structures, 41, 1025-1037.

Daniel, J. S., and Kim, Y. R., 2002. Development of a simplified fatigue test and analysis procedure using a viscoelastic, continuum damage model. Journal of the Association of Asphalt Paving Technologists, 71, 619-650.

Darabi, M. K., et al., 2011. A thermo-viscoelastic-viscoplastic-viscodamage constitutive model for asphaltic materials. International Journal of Solids and Structures, 48, 191-207.

Daux, C., et al., 2000. Arbitrary branched and intersecting cracks with the extended finite element method. International Journal for Numerical Methods in Engineering, 48 (12), 1741-1760.

Duarte, C. A., Babuska, I., and Oden, J. T., 2000. Generalized finite element methods for threedimensional structural mechanics problems. Computers and Structures, 77 (2), 215-232.

Duarte, C. A., and Oden, J. T., 1996. An h-p adaptive method using clouds. Computer Methods in Applied Mechanics and Engineering, 139 (1-4), 237-262.

Dugdale, D., 1960. Yielding of steel sheets containing slits. Journal of Mechanics and Physics of Solids, 8, 100-104.

Espinosa, H. D., Dwivedi, S., and Lu, H. C., 2000. Modeling impact induced delamination of woven fiber reinforced composites with contact/cohesive laws. Computer Methods in Applied Mechanics and Engineering, 183 (3-4), 259-290.

Espinosa, H. D., and Zavattieri, P. D., 2003. A grain level model for the study of failure initiation and evolution in polycrystalline brittle materials, part I: theory and numerical implementation. Mechanics of Materials, 35 (3-6), 333-364.

Feng, Z., et al., 2008. Virtual testing procedure for cracking performance prediction of HMA. Proceedings of the sixth RILEM International Conference on Cracking in Pavements. Chicago, IL: CRC Press, 575-585.

Feyel, F., 1999. Multiscale $\mathrm{FE}^{2}$ elastoviscoplastic analysis of composite structures. Computational Materials Science, 16, 344-354.

Feyel, F., and Chaboche, J. L., 2000. FE² multiscale approach for modeling the elastoviscoplastic behavior of long fibre $\mathrm{SiC} / \mathrm{Ti}$ composite materials. Computer Methods in Applied Mechanics and Engineering, 183, 309-330.

Fish, J., and Belsky, V., 1995. Multigrid method for periodic heterogeneous media, part II: multiscale modeling and quality control in multidimensional cases. Computer Methods in Applied Mechanics and Engineering, 126, 17-38.

Fish, J., and Shek, K., 2000. Multiscale analysis of composite materials and structures. Composites Science and Technology, 60, 2547-2556.

Fish, J., and Wagiman, A., 1993. Multiscale finite element method for a locally non-periodic heterogeneous medium. Computational Mechanics, 12, 164-180.

Geubelle, P. H., and Baylor, J. S., 1998. Impact-induced delamination of composites: a 2D simulation. Composites Part B: Engineering, 29 (5), 589-602.

Ghosh, S., Lee, K., and Raghavan, P., 2001. A multi-level computational model for multiscale damage analysis in composite and porous materials. International Journal of Solids and Structures, 38, 23352385.

Gibson, N. H., et al., 2003. Viscoelastic, viscoplastic, and damage modeling of asphalt concrete in unconfined compression. Transportation Research Record, 1860, 3-15. 
Griffith, A. A., 1920. The phenomena of rupture and flow in solids. Philosophical Transactions - Series A, 221, 163-198.

Gustafsson, P. J., 1985. Fracture mechanics studies of nonyielding materials like concrete: modeling of tensile fracture and applied strength analyses. Report no. TVBM-1007, Division of Building Materials, Lund Institute of Technology, Lund, Sweden.

Haj-Ali, R. M., and Muliana, A. H., 2004. A multi-scale constitutive formulation for the nonlinear viscoelastic analysis of laminated composite materials and structures. International Journal of Solids and Structures, 41, 3461-3490.

Hillerborg, A., 1985. The theoretical basis of a method to determine the fracture energy GF of concrete. Materials and Structures, 18, 291-296.

Hillerborg, A., Modeer, M., and Petersson, P. E., 1976. Analysis of crack formation and crack growth in concrete by means of fracture mechanics and finite elements. Cement and Concrete Research, 6 (6), 773-781.

Jeng, Y. S., Liaw, C., and Liu, P., 1993. Analysis of crack resistance of asphalt concrete overlays - a fracture mechanics approach. Transportation Research Record, 1388, 160-166.

Jeng, Y. S., and Perng, J. D., 1991. Analysis of crack propagation in asphalt concrete using cohesive crack model. Transportation Research Record, 1317, 90-99.

Khalid, H. A., and Artamendi, I., 2008. Measurement and effective evaluation of crack growth in asphalt mixtures. Proceedings of the sixth RILEM international conference on cracking in pavements, Chicago, IL: CRC Press, 417-425.

Kim, Y., Allen, D. H., and Little, D. N., 2005. Damage-induced modeling of asphalt mixtures through computational micromechanics and cohesive zone fracture. Journal of Materials in Civil Engineering, 17 (5), 477-484.

Kim, Y., Allen, D. H., and Little, D. N., 2006. Computational model to predict fatigue damage behavior of asphalt mixtures under cyclic loading. Transportation Research Record, 1970, 196-206.

Kim, Y., Allen, D. H., and Little, D. N., 2007. Computational constitutive model for predicting nonlinear viscoelastic damage and fracture failure of asphalt concrete mixtures. International Journal of Geomechanics, 7 (2), 102-110.

Kim, Y., et al., 2010a. Damage modeling of bituminous mixtures considering mixture microstructure, viscoelasticity, and cohesive zone fracture. Canadian Journal of Civil Engineering, 37, 1125-1136.

Kim, H., and Buttlar, W. G., 2009. Finite element cohesive fracture modeling of airport pavements at low temperatures. Cold Regions Science and Technology, 57, 123-130.

Kim, Y. R., and Little, D. N., 1990. One-dimensional constitutive modeling of asphalt concrete. Journal of Engineering Mechanics, 116 (4), 751-772.

Kim, Y., and Lutif, J., 2008. Computational micromechanics modeling for damage-induced behavior of asphalt mixtures considering viscoelasticity and cohesive zone fracture. In: Z. You, A. R. Abbas, and L. Wang, eds. ASCE Geotechnical Special Publication: Pavements and Materials-Modeling, Testing, and Performance, ASCE, 17-25.

Kim, Y., Souza, F., and Little, D.N., 2010b. Multiscale modeling of bituminous mixtures considering material viscoelasticity and cohesive zone fracture. In: A.R. Tarefder, Y. Kim, Z. You and L. Wang, eds. ASCE Engineering Mechanics Special Publication: Pavements and Materials - Testing and Modeling in Multiple Length Scales, ASCE, 74-85.

Kim, H., Wagoner, M. P., and Buttlar, W. G., 2009. Micromechanical fracture modeling of asphalt concrete using a single-edge notched beam test. Materials and Structures, 42, 677-689.

Klein, P., et al., 2001. Physics-based modeling of brittle fracture: cohesive formulations and the application of mesh-free methods. Technical Report SAND2001-8099, Sandia National Laboratories. 
Knauss, W. G., 1970. Delayed failure - the Griffith problem for linearly viscoelastic materials. International Journal of Fracture, 6 (1), 7-20.

Lee, H. J., Daniel, J. S., and Kim, Y. R., 2000. Continuum damage mechanics-based fatigue model of asphalt concrete. Journal of Materials in Civil Engineering, 12 (2), 105-112.

Lee, H. J., Kim, Y. R., and Lee, S. W., 2003. Prediction of asphalt mix fatigue life with viscoelastic material properties. Transportation Research Record, 1832, 139-147.

Lee, K., Moorthy, S., and Ghosh, S., 1999. Multiple scale computational model for damage in composite materials. Computer Methods in Applied Mechanics and Engineering, 172, 175-201.

$\mathrm{Li}, \mathrm{X}$. , 2005. Investigation of the fracture resistance of asphalt mixtures at low temperature with a semi-circular bend (SCB) test. Dissertation (PhD). University of Minnesota, Minneapolis, MN.

Li, X., et al., 2005. Prediction of low temperature crack spacing in asphalt pavements. Proceedings of the Seventh International Conference on the Bearing Capacity of Roads, Railways, and Airfields, Trondheim, Norway: Norwegian University of Science and Technology.

Lutif, J., et al., 2010. Multiscale modeling to predict the mechanical behavior of asphalt mixtures. Transportation Research Record, 2181, 28-35.

Majidzadeh, K., Kaufmann, E. M., and Ramsamooj, D. V., 1971. Application of fracture mechanics in the analysis of pavement fatigue. Proceedings of the Association of Asphalt Pavement Technologists, Vol. 40. AAPT, 227-246.

Marasteanu, M. O., et al., 2002. Determining the low-temperature fracture toughness of asphalt mixtures. Transportation Research Record, 1789, 191-199.

Masad, E., et al., 2001. Modeling and experimental measurements of localized strain distribution in asphalt mixes. Journal of Transportation Engineering, 127 (6), 477-485.

Masad, E., et al., 2005. Viscoplastic modeling of asphalt mixes with the effects of anisotropy, damage, and aggregate characteristics. Mechanics of Materials, 37 (12), 1242-1256.

Miller, O., Freund, L., and Needleman, A., 1999. Modeling and simulation of dynamic fragmentation in brittle materials. International Journal of Fracture, 96 (2), 101-125.

Mobasher, B. M., Mamlouk, M. S., and Lin, H. M., 1997. Evaluation of crack propagation properties of asphalt mixtures. Journal of Transportation Engineering, 123 (5), 405-413.

Moes, N., and Belytschko, T., 2002. Extended finite element method for cohesive crack growth. Engineering Fracture Mechanics, 69 (7), 813-833.

Mohammad, L. N., and Kabir, M. D., 2008. Evaluation of fracture properties of hot mix asphalt. Pavement Cracking: Mechanisms, Modeling, Detection, Testing, and Case Histories, CRC Press, 427-436.

Molenaar, J. M. M., and Molenaar, A. A. A., 2000. Fracture toughness of asphalt in the semi-circular bend test. Proceedings of the second Eurasphalt and Eurobitume Congress, Barcelona, Spain: Foundation Eurasphalt.

Mota, A., Knap, J., and Ortiz, M., 2008. Fracture and fragmentation of simplicial finite element meshes using graphs. International Journal for Numerical Methods and Engineering, 73, 1547-1570.

Needleman, A., 1987. A continuum model for void nucleation by inclusion debonding. Journal of Applied Mechanics - Transactions of the ASME, 54 (3), 525-531.

Needleman, A., 1990. An analysis of tensile decohesion along an interface. Journal of the Mechanics and Physics of Solids, 38 (3), 289-324.

Oden, J. T., Vemaganti, K., and Moes, N., 1999. Hierarchical modeling of heterogeneous solids. Computer Methods in Applied Mechanics and Engineering, 148, 367-391.

Oden, J. T., and Zohdi, T. I., 1997. Analysis and adaptive modeling of highly heterogeneous elastic structures. Computer Methods in Applied Mechanics and Engineering, 172, 3-25. 
Ortiz, M., and Pandolfi, A., 1999. Finite-deformation irreversible cohesive elements for three-dimensional crack propagation analysis. International Journal for Numerical Methods in Engineering, 44 (9), 12671282.

Pandolfi, A., and Ortiz, M., 1998. Solid modeling aspects of three-dimensional fragmentation. Engineering with Computers, 14 (4), 287-308.

Pandolfi, A., and Ortiz, M., 2002. An efficient adaptive procedure for three-dimensional fragmentation simulations. Engineering with Computers, 18, 148-159.

Papagiannakis, A. T., Abbas, A., and Masad, E., 2002. Micromechanical analysis of viscoelastic properties of asphalt concretes. Transportation Research Record, 1789, 113-120.

Papoulia, K. D., Sam, C. H., and Vavasis, S. A., 2003. Time continuity in cohesive finite element modeling. International Journal for Numerical Methods in Engineering, 58, 679-701.

Park, K., 2009. Potential-based fracture mechanics using cohesive zone and virtual internal bond modeling. Dissertation (PhD). University of Illinois, Urbana Champaign, IL.

Park, K., Paulino, G. H., and Roesler, J. R., 2009. A unified potential-based cohesive model of mixedmode fracture. Journal of the Mechanics and Physics of Solids, 57 (6), 891-908.

Park, S. W., Kim, Y. R., and Schapery, R. A., 1996. A viscoelastic continuum damage model and its application to uniaxial behavior of asphalt concrete. Mechanics of Materials, 24, 241-255.

Paulino, G. H., et al., 2008. A general topology-based framework for adaptive insertion of cohesive elements in finite element meshes. Engineering with Computers, 24 (1), 59-78.

Raghavan, P., et al., 2001. Revisiting the composite laminate problem with an adaptive multi-level computational model. Composites Science and Technology, 61, 1017-1040.

Rice, J. R., and Wang, J. S., 1989. Embrittlement of interfaces by solute segregation. Material Science and Engineering, 107, 23-40.

Ruiz, G., Pandolfi, A., and Ortiz, M., 2001. Three-dimensional cohesive modeling of dynamic mixedmode fracture. International Journal for Numerical Methods in Engineering, 52 (1-2), 97-120.

Sadd, M. H., et al., 2003. Simulation of asphalt materials using a finite element micromechanical model with damage mechanics. Transportation Research Record, 1832, 86-95.

Sam, C. H., Papoulia, K. D., and Vavasis, S. A., 2005. Obtaining initially rigid cohesive finite element models that are temporarily convergent. Engineering Fracture Mechanics, 72, 2247-2267.

Scheider, I., and Brocks, W., 2003. Simulation of cup-cone fracture using the cohesive model. Engineering Fracture Mechanics, 70, 1943-1961.

Shet, C., and Chandra, N., 2002. Analysis of energy balance when using cohesive zone models to simulate fracture processes. Journal of Engineering Materials and Technology - Transactions of the ASME, 124, 440-450.

Shim, D.-J., Paulino, G. H., and Dodds, R. H., Jr., 2006. J resistance behavior in functionally graded materials using cohesive zone and modified boundary layer models. International Journal of Fracture, 139 (1), 91-117.

Soares, B. J., Freitas, F., and Allen, D. H., 2003. Crack modeling of asphaltic mixtures considering heterogeneity of the material. Transportation Research Record, 1832, 113-120.

Soares, R., Kim, Y., and Allen, D. H., 2008. Multiscale computational modeling for predicting evolution of damage in asphaltic pavements. Proceedings of the Sixth RILEM International Conference on Cracking in Pavements, Chicago, IL: CRC Press, 599-608.

Soares, R., Kim, Y., and Allen, D. H., 2009. FEM multiscale analysis of pavement structure including damage. Proceedings of the Fourth Biot Conference on Poromechanics, Columbia University, NY: DEStech Publications, Inc., 517-522. 
Song, S. H., Paulino, G. H., and Buttlar, W. G., 2006a. A bilinear cohesive zone model tailored for fracture of asphalt concrete considering viscoelastic bulk material. Engineering Fracture Mechanics, 73, 2829-2847.

Song, S. H., Paulino, G. H., and Buttlat, W. G., 2006b. Simulation of crack propagation in asphalt concrete using an intrinsic cohesive zone model. Journal of Engineering Mechanics, 132 (11), 12151223.

Souza, F. V., and Allen, D. H., 2010. Multiscale modeling of impact on heterogeneous viscoelastic solids containing evolving microcracks. International Journal for Numerical Methods in Engineering, 82, 464-504.

Souza, F. V., et al., 2004. Model for predicting damage evolution in heterogeneous viscoelastic asphaltic mixtures. Transportation Research Record, 1891, 131-139.

Tashman, L., et al., 2004. Damage evolution in triaxial compression tests of HMA at high temperatures. Journal of the Association of Asphalt Paving Technologists, 73, 53-87.

Tvergaard, V., 1990. Effect of fiber debonding in a whisker-reinforced metal. Materials Science and Engineering, A125, 203-213.

Tvergaard, V., and Hutchinson, J. W., 1992. The relation between crack growth resistance and fracture process parameters in elastic-plastic solids. Journal of the Mechanics and Physics of Solids, 40 (6), 1377-1397.

Tvergaard, V., and Hutchinson, J. W., 1993. The influence of plasticity on mixed mode interface toughness. Journal of the Mechanics and Physics of Solids, 41 (6), 1119-1135.

van Rooijen, R. C., and de Bondt, A. H., 2008. Crack propagation performance evaluation of asphaltic mixes using a new procedure based on cyclic semi-circular bending tests. Proceedings of the Sixth RILEM International Conference on Cracking in Pavements, Chicago, IL: CRC Press, 437-446.

Wagoner, M. P., Buttlar, W. G., and Paulino, G. H., 2005a. Development of a single-edge notched beam test for asphalt concrete mixtures. Journal of Testing and Evaluation, 33 (6), 452-460.

Wagoner, M. P., Buttlar, W. G., and Paulino, G. H., 2005b. Disk-shaped compact tension test for asphalt concrete fracture. Society for Experimental Mechanics, 45 (3), 270-277.

Wagoner, M. P., et al., 2005c. Investigation of the fracture resistance of hot-mix asphalt concrete using a disk-shaped compact tension test. Transportation Research Record, 1929, 183-192.

Wells, G. N., and Sluys, L. J., 2001. A new method for modelling cohesive cracks using finite elements. International Journal for Numerical Methods in Engineering, 50 (12), 2667-2682.

Xu, X. P., and Needleman, A., 1993. Void nucleation by inclusion debonding in a crystal matrix. Modelling and Simulation in Materials Science and Engineering, 1 (2), 111-132.

Xu, X. P., and Needleman, A., 1994. Numerical simulation of fast crack growth in brittle solids. Journal of Mechanics and Physics of Solids, 42 (9), 1397-1434.

Yang, Q. D., and Thouless, M. D., 2001. Mixed-mode fracture analyses of plastically deforming adhesive joints. International Journal of Fracture, 110 (2), 175-187.

Yoon, C., and Allen, D. H., 1999. Damage dependent constitutive behavior and energy release rate for a cohesive zone in a thermoviscoelastic solid. International Journal of Fracture, 96 (1), 55-74.

You, Z., Adhikari, S., and Kutay, M. E., 2009. Dynamic modulus simulation of the asphalt concrete using the X-ray computed tomography images. Materials and Structures, 42, 617-630.

You, Z., and Buttlar, W. G., 2004. Discrete element method to predict the modulus of asphalt concrete mixtures. Journal of Materials in Civil Engineering, 16 (2), 140-146. 
You, Z., and Buttlar, W. G., 2005. Application of discrete element modeling techniques to predict the complex modulus of asphalt-aggregate hollow cylinders subjected to internal pressure. Transportation Research Record, 1929, 218-226.

You, Z., and Buttlar, W. G., 2006. Micromechanical modeling approach to predict compressive dynamic moduli of asphalt mixture using the distinct element method. Transportation Research Record, 1970, 73-83.

Zavattieri, P. D., and Espinosa, H. D., 2001. Grain level analysis of crack initiation and propagation in brittle materials. Acta Materialia, 49 (20), 4291-4311.

Zhang, Z., and Paulino, G. H., 2005. Cohesive zone modeling of dynamic failure in homogeneous and functionally graded materials. International Journal of Plasticity, 21 (6), 1195-1254.

Zhou, F., and Molinari, J. F., 2004. Dynamic crack propagation with cohesive elements: a methodology to address mesh dependency. International Journal for Numerical Methods in Engineering, 59, 1-24. 